\title{
¡Mira qué alegría! Promoviendo el conocimiento de la cultura andaluza en Educación Secundaria Obligatoria: una propuesta didáctica'
}

\author{
What a Joy! Promoting the Cultural Knowledge of the Spanish Region \\ of Andalusia in the Secondary Obligatory Education: \\ An Educational Proposal
}

\author{
ESTER TRIGO IBÁÑEZ \\ IRENE MARÍA RODRÍGUEZ BLÁZQUEZ \\ PABLO MORENO VERDULLA \\ Universidad de Cádiz \\ Centro del Profesorado de Cádiz \\ IES Ciudad de Hércules \\ España \\ ester.trigo@uca.es \\ irene.rodriguez.b.edu@juntadeandalucia.es \\ pmverdulla@gmail.com
}

(Recibido: 22-OI-2OI8; aceptado:3-08-20I8)

\footnotetext{
I Para citar este artículo: Trigo Ibánez, Ester; Rodríguez Blázquez, Irene Ma y Moreno Verdulla, Pablo (20r9). ¡Mira qué alegría! Promoviendo el conocimiento de la cultura andaluza en Educación Secundaria Obligatoria: una propuesta didáctica. Alabe 19. [www.revistaalabe.com]

DOI: IO.I5645/Alabe20i9.19.7
} 
Resumen. Una de las premisas básicas de la concreción que se hace en Andalucía del currículum es la presencia de elementos referidos al patrimonio cultural de la comunidad autónoma. El flamenco es una pieza fundamental dentro de la cultura andaluza y, por ello, la Administración pone a disposición del profesorado una serie de recursos para garantizar la presencia de esta manifestación folkórica en las aulas andaluzas. Situados en la didáctica del flamenco, en este trabajo ofrecemos la planificación de un proyecto interdisciplinar -desde la literatura a la música- que, a través de la indagación sobre el concepto de alegrías de Cádiz, permitirá desarrollar las siete competencias clave desde una perspectiva funcional.

Palabras clave: Didáctica de la Literatura; flamenco; competencias clave; cultura andaluza; aprendizaje basado en proyectos.
Abstract. One of the basic premises of the concretion that is made in Andalusia of the curriculum is the presence of elements inserted in the cultural heritage of the Andalusian citizenship. Since flamenco is a fundamental piece within the Andalusian culture, the administration makes available for teachers a series of resources in order to guarantee the presence of this folkloric manifestation in Andalusian schools. Located in the didactics of flamenco, in this paper we offer an interdisciplinary Project from Literature to Music. Through the research on the concept of Alegrías de Cádiz the seven key competences will be developed from a functional perspective.

Keywords: Didactics of Flamenco; key competences;Andalusian culture; project-based learning 
Pero le sobran con tres

Cinco letras tiene Cai

Pero le sobran con tres

Que en ellas cabe la gracia

al derecho y al revés

Que en ellas cabe la gracia

Alderecho y al revés

\section{Introducción}

El folklore, en general, resulta de la interacción entre el ser humano y el medio en que habita; el cante flamenco, en particular, constituye una manifestación folklórica en la que la ciudadanía andaluza se ha visto inmersa desde tiempos inmemoriales. De acuerdo con esta idea, Rossy (ig66) afirma que el ejercicio profesional del flamenco se remonta a los tiempos del imperio romano, concretamente a los siglos I y II, momento en que bailarinas y cantantes gaditanos eran contratados por grupos, constituyendo lo que en la actualidad se conoce como cuadros flamencos.

En Andalucía, hablar de folklore supone remitirnos obligatoriamente al cante flamenco y a la poesía tradicional y popular que se ha mantenido viva en su dimensión formal y temática gracias a la promoción internacional que ha ido adquiriendo paulatinamente (Schuchardt, I990; Cruces, 2003). No en vano, en noviembre de 20I0, a petición de tres Comunidades Autónomas -Andalucía, Extremadura y Murcia-, el flamenco fue declarado patrimonio cultural inmaterial de la humanidad por la UNESCO².

La presencia del flamenco en Andalucía no es casual, sino que se debe, en gran parte, a la interacción de la cultura gitana con los moriscos residentes en el Reino de Granada. Así, como afirma Fernández-Fígares (I995: 28) “en ningún país del mundo los gitanos practican este arte más que en Andalucía, donde la saga de los maestros consumados, tanto del cante como del baile, de raza gitana, es amplísima”.

Tanto el cante como el baile flamenco, independientemente del palo en el que se enmarquen, expresan toda una gama de emociones y estados de ánimo -alegría, tristeza, temor, desamor, regocijo...- El cante lo hace a través del acompañamiento musical, el tono, el ritmo y la palabra -sencilla, directa y concisa- y el baile, a través de los movimientos corporales de bailaores y bailaoras. No obstante, esta expresión artística no sería posible sin la interrelación de manifestaciones culturales reflejadas en la literatura, la música y la danza.

\footnotetext{
${ }^{2}$ Para mayor información, puede consultarse: https://goo.gl/aEwmqS
} 
Uno de los cantes gaditanos más conocidos son las alegrías ${ }^{3}$. Se localizan principalmente en Cádiz capital. Su estructura es sencilla y fácilmente comprensible. Consta de coplas romanceadas que se ajustan al compás de la soleá, pero con mayor viveza. Normalmente son bailadas por una mujer. Desde el plano formal, como todos los palos del flamenco, las alegrías presentan métricas variables, cuestión que plantea problemas rítmicos a la hora de la interpretación musical. Generalmente, estos son solventados gracias a la pericia de los maestros de la guitarra que acompaña a los cantaores y a las cantaoras, alargando o acortando los compases para adaptarse a la letra y al ritmo de sus intérpretes. En cuanto a la temática, las alegrías hablan sobre Cádiz y todos los pueblos de su bahía, el mar, el flamenco, Andalucía, el amor y el desamor (Ropero, I984).

En el ámbito de la investigación didáctica, las propuestas para trabajar el flamenco en las aulas vienen realizándose desde hace años (Navarro, ı986; Sánchez y Navarro, I988; León, I99O; López, 2004; Grande, 2005; Utrilla, 2007; Delgado, 2OII). Estas propuestas persiguen, tal y como señala López (2OIO: 2) "que toda la población conozca el flamenco por sus valores culturales, históricos, sociológicos, antropológicos, artísticos, etc.”. Para estos autores, la escuela constituye un lugar ideal para el desarrollo de una didáctica del flamenco, tanto desde el área de Lengua Castellana y Literatura como desde el área de Música. En ambos casos el flamenco supone un recurso de alto valor para desarrollar la educación literaria y el acercamiento a esta manifestación folklórica. En consonancia con esta idea, Perea (2OI4) creó un blog en el que se compilan materiales didácticos y experiencias para llevar el flamenco al aula de Educación Primaria. Sin embargo, aunque las propuestas se realicen para la etapa primaria, son amplias las posibilidades para adaptarlas al marco de la Educación Secundaria.

Desde nuestro punto de vista, coincidiendo con Julio (20I2) y con Gutiérrez y Perea (2OI5), la didáctica del flamenco debe abordarse desde la interdisciplinariedad, es decir, partir de la asignatura Lengua castellana y literatura, desde la poesía popular como hecho literario y cultural (Uccella, 2OI3) y profundizar en la asignatura de Música con la audición y representación del hecho flamenco (Gutiérrez, I99O; De Vicente, 20I3).

A lo largo de estas páginas presentamos un proyecto de trabajo donde se interrelacionan dos áreas -la literatura y la música- y cuyo objetivo principal será el conocimiento, la composición e interpretación rítmica de alegrías de Cádiz por parte de estudiantes de primer curso de la Educación Secundaria Obligatoria (en adelante, ESO).

Además, dado que se considera que las alegrías de Cádiz surgieron del contacto entre los aragoneses y los gaditanos en la Guerra de la Independencia, nuestra propuesta cobra sentido si la planificamos desde una propuesta educativa referida a dos centros escolares: uno, situado en la provincia de Cádiz; y otro, en la Comunidad Autónoma de Aragón. Así, se planteará a cada centro el reto de crear y filmar un documental que recoja toda la información posible sobre ambas manifestaciones folklóricas: la alegría y la jota.

\footnotetext{
3 Puede encontrarse amplia información sobre el origen de las alegrías de Cádiz en: https://goo.gl/cA87hQ

${ }^{4}$ http://flamencoprimaria.blogspot.com.es/
} 
Los gaditanos centrarán su atención en las alegrías de Cádiz y los aragoneses, en la jota aragonesa. Tras la creación del documental, se intercambiarán los productos para que el aprovechamiento de la experiencia didáctica sea completo.

En este sentido, hemos elegido la metodología del aprendizaje basado en proyectos (en adelante, $\mathrm{ABP}$ ) al considerarla idónea para el desarrollo de las competencias clave (Romero, Heredia y Ordóñez, 2OI7). De acuerdo con esta idea, coincidimos plenamente con los planteamientos de Trujillo (20I6: г3):

hay maneras de enseñar que entienden que aprender no es sólo aprender y memorizar, sino también buscar, elegir, discutir, aplicar, errar, corregir, ensayar. Hay maneras de enseñar que demuestran que 'aprender' puede ser una modalidad de 'hacer'. Y maneras de enseñar que, está demostrado científicamente, no son efectivas para que los alumnos aprendan.

Así, para escribir alegrías y preparar un documental, los estudiantes deberán buscar información sobre este palo flamenco, observar modelos, discutir sobre la temática que tratarán, crear textos, tomar decisiones sobre el orden en el que filmarán cada secuencia, los elementos que incluirán, el storyboard 5 ... Todos estos procesos harán de nuestra propuesta un modelo funcional que permitirá a los estudiantes desarrollar las competencias clave y activar sus conocimientos (Zabala y Arnau, 2OO7; 2OI4; Puig y Martín, 2OO7; Escamilla, 2009; Feito, 20IO; Lomas, 2OIO; Majó, 2OIO).

En la programación de nuestro proyecto, como desarrollaremos detenidamente más adelante, hemos seguido la siguiente secuencia: presentación, que servirá como un momento para motivar al alumnado; comprensión, donde se organizarán talleres para recabar información sobre el tema objeto de estudio; práctica, fase en la que el alumnado realizará ejercicios y actividades que le ayudarán a alcanzar con éxito la tarea final; transferencia, será un momento crucial, pues servirá para preparar la tarea final, en nuestro caso, el documental y evaluación, donde se mostrarán los documentales de cada grupo y se hará una dinámica de co-evaluación.

\footnotetext{
5 El Storyboard -o guion gráfico- es un conjunto de ilustraciones mostradas secuencialmente que persigue servir de guía para entender una historia o seguir la estructura de una película, un documental o un cómic antes de dibujarse o filmarse. Este modo de previsualización es muy utilizado en la industria cinematográfica.
} 


\section{La presencia del flamenco en el currículum}

Como señalábamos con anterioridad, es amplio el número de docentes e investigadores que vienen reivindicando desde los años 8o del siglo pasado una didáctica del flamenco. Algunos autores, como López (2OIO), si bien reconocen que las Administraciones, especialmente la Junta de Andalucía, han incorporado en sus concreciones curriculares el estudio del flamenco desde diversas perspectivas, también señalan que aún es necesario continuar reclamando la presencia de este arte desde la formación inicial y la permanente del profesorado.

Para determinar la presencia del flamenco en el currículum es necesario partir de la normativa de aplicación nacional y observar la concreción que la administración andaluza ha realizado desde 20I4 hasta la actualidad. En la tabla i se reflejan los referentes legales que justifican el tratamiento del flamenco en las aulas andaluzas:

Tabla 1

Referentes que justifican el tratamiento del flamenco en las aulas andaluzas

\begin{tabular}{ll}
\hline LOCALIZACIÓN & REFERENCIA LEGAL \\
& TERRITORIO DE \\
APLICACIÓN
\end{tabular}

R.D. 1105/2014, de 26 de diciembre, por

BOE el que se establece el currículo básico de la Educación Secundaria Obligatoria y del NACIONAL Bachillerato.

Orden de 14 de julio de 2016, por la que se desarrolla el currículo correspondiente a la Educación Secundaria Obligatoria en la BOJA Comunidad Autónoma de Andalucía, se regulan determinados aspectos de la atención a la diversidad y se establece la ordenación ANDALUZA de la evaluación del proceso de aprendizaje del alumnado.

Orden de 7 de mayo de 2014, por la que se BOJA establecen medidas para la inclusión del Flamenco en el sistema educativo andaluz. 
a) En el R. D. IIO5/20I4 encontramos referencias -contenidos, criterios de evaluación y estándares de aprendizaje - al flamenco como manifestación artística en cuatro asignaturas específicas, como son:

- Artes escénicas y danza, de $4 \cdot^{\circ}$ curso de ESO, cuyo bloque 3 está dedicado a la danza. Para la correcta adquisición de los contenidos, los estudiantes deben explicar, reconocer, clasificar y situar en el tiempo los diferentes estilos de danza, entre los que se incluye el flamenco, partir de modelos visionados en el aula.

- Lenguaje y práctica musical, de r. ${ }^{\circ}$ de Bachillerato. Esta asignatura dedica el bloque 2 a la audición comprensiva. Para superarla, los estudiantes deben, partiendo de audiciones realizadas en clase, reconocer auditivamente y determinar las características de las obras escuchadas o interpretadas. Los estilos abordados son la música culta, el jazz, el rock, el flamenco, así como los más importantes del lenguaje musical contemporáneo.

- Fundamentos del arte II, de $2 .{ }^{\circ}$ curso de Bachillerato, cuyo bloque Io aborda el auge del flamenco y las figuras de Paco de Lucía, Camarón de la Isla, Carmen Amaya y Antonio Gades. El estudio de estos contenidos está orientado a que los estudiantes identifiquen la obra de los distintos autores, expliquen las claves del éxito internacional del flamenco, reconozcan la importancia de la danza flamenca en el mundo y, finalmente, sean capaces de ubicar el flamenco en España y de extraer conclusiones a través de los datos obtenidos.

- Análisis musical, también de $2 .^{\circ}$ curso de Bachillerato, cuyo bloque in se dedica a distintos estilos musicales, como el jazz, la música urbana y el flamenco. En esta asignatura se pretende que los estudiantes distingan y analicen las características musicales y sociológicas de los diferentes estilos musicales estudiados conceptualmente.

b) El flamenco, como se podría esperar dada la importancia que tiene en la realidad cultural andaluza, está mucho más presente en la Orden de I4 de julio de 20I6, desde los objetivos hasta las áreas troncales: Lengua castellana y literatura y Música, pasando por las recomendaciones metodológicas de asignaturas troncales y específicas.

- Objetivos. El objetivo ‘b’ hace referencia específica al conocimiento y aprecio de la cultura andaluza "para que sea valorada y respetada como patrimonio propio y en el marco de la cultura española y universal”. El flamenco es uno de los elementos patrimoniales de la Comunidad Autónoma de Andalucía, por lo que será prioritario para los docentes fomentar su conocimiento y aprecio.

- Estrategias metodológicas para Lengua castellana y literatura. En este apartado se hace referencia explícita a los programas específicos para promover la educación lingüística y literaria desde una perspectiva comunicativa y competencial. Además de ello, se detallan los recursos que la Consejería de Educación pone a disposición del profesorado a través de la red telemática Averroes. Esta red contiene enlaces a interesantes e innovadores blogs sobre flamenco y a diferentes portales y webs bastante útiles para el profesorado como, por ejemplo, Flamencoeduca, que es un portal educativo plagado de recursos.

- Estrategias metodológicas para Artes escénicas y danza. En esta asignatura se hace especial hincapié en el trabajo del legado cultural andaluz utilizando recursos como 
"alegorías de poetas andaluces que se integran en la danza flamenca, el teatro Lorquiano, la pluma modernista de Machado o Juan Ramón Jiménez, raíces de la esencia de la fusión dramática que unos martinetes puedan generar coreográficamente, soleares con fusión contemporánea en la danza con la raíz del baile flamenco, performance ars o baile contemporáneo, tanto en el teatro como en la dramatización de poemas".

- Lengua castellana y literatura. El flamenco está presente en todos los cursos de la ESO dentro del bloque i, referido a la Comunicación oral. A través del estudio del flamenco en esta asignatura, se espera que los estudiantes sean capaces de reconocer y respetar la riqueza y variedad de las hablas existentes en Andalucía; memorizar y recitar textos orales desde el conocimiento de sus rasgos estructurales y de contenido; y de reconocer las características de la modalidad lingüística andaluza en diferentes manifestaciones orales.

- Música. Esta es la asignatura que más se centra en el estudio del flamenco: los principales artistas, los distintos palos y estilos... Este contenido se aborda desde todos los bloques: Interpretación y creación; Escucha; Contextos musicales y culturales; y Música y tecnología. Se destaca el flamenco como género de música y danza "que se originó y desarrolló en la Baja Andalucía a partir del Siglo XIX, y que actualmente es considerado Patrimonio Inmaterial de la Humanidad”. A través de esta asignatura se pretende, entre otras cuestiones, que la ciudadanía andaluza conozca las raíces del flamenco e identifique sus principales palos comprendiendo y apreciando la variedad lingüística andaluza y la importancia del flamenco dentro de la literatura andaluza.

c) Pero no es hasta la Orden de 7 de mayo de $20{ }_{4} 4$ cuando se establecen medidas para la inclusión del Flamenco en el sistema educativo andaluz. En ella se crea el Portal Educativo del Flamenco, incluyéndolo dentro de la planificación de actividades complementarias y extraescolares de los centros y se establece la convocatoria de proyectos de investigación e innovación y desarrollo curricular y de elaboración de materiales curriculares y recursos didácticos para la inclusión del flamenco en el sistema educativo andaluz.

d) Finalmente, las Instrucciones de 6 de noviembre de 20I4 para la celebración del Día del Flamenco, que se conmemora el día i6 de noviembre, determinan que los centros educativos andaluces deberán programar actividades orientadas a promover el conocimiento y la reflexión en torno al flamenco como elemento singular del patrimonio cultural andaluz.

\section{3• ¡Mira qué alegría! Una propuesta didáctica interdisciplinar}

Como se aprecia en el análisis curricular, la administración andaluza apuesta por la inclusión del flamenco en las aulas como elemento singular del patrimonio cultural andaluz. No solo la presencia de esta manifestación artística es mayor en la concreción curricular para Andalucía, sino que, por una parte, se amplía el abanico de áreas que se acercan al flamenco y, por otra, se pone a disposición del profesorado una serie de recursos de apoyo 
para elaborar materiales, programar actividades complementarias y extraescolares, etc.

Dado que, como se ha podido constatar, las escuelas andaluzas pueden realizar propuestas para la promoción y el conocimiento del flamenco desde distintas áreas y perspectivas didácticas, nuestro trabajo consiste en un proyecto, titulado ¡Mira qué alegría! que implica a las áreas de Lengua Castellana y Literatura y Música.

Hemos optado por situar nuestra propuesta en I ${ }^{\circ}$ ESO puesto que el alumnado posee el andamiaje apropiado para activar conocimientos que le llevarán a componer textos poéticos sencillos, mantener el ritmo con palmas, procesar la información a partir de entornos virtuales, realizar pequeñas búsquedas, manejar dispositivos electrónicos, etc. (Ambròs y Ramos, 2007; Corpas, 20I7). Los elementos curriculares necesarios para el correcto desempeño de este proyecto aparecen detallados en el Anexo I: Planificación del proyecto ¡Mira quéalegría!

Como anticipábamos en la introducción, la secuencia didáctica responde a cinco fases, que pasamos a detallar:

\section{I. Fase I: Presentación}

Tendrá lugar en la asignatura de Lengua Castellana y Literatura. Esta fase ocupará la primera sesión y servirá como elemento motivador. Al inicio de la clase se proyectará un vídeo en el que un docente de un centro aragonés establece contacto con los alumnos y las alumnas del grupo y les pide ayuda: ha sabido que en Cádiz existe un cante flamenco que guarda relación con la jota aragonesa y quisiera obtener más información para mostrársela a sus estudiantes. Además de solicitarles ayuda, les promete que su clase, también de primer curso de ESO, les hará partícipe de su folklore regional.

Tras el visionado, se propiciará un debate sobre las variedades de habla en España en general y el habla de Cádiz en particular. Se irán tomando notas en la pizarra con el fin de que el alumnado las utilice como base para realizar una práctica letrada: la escritura de un texto sobre la variedad lingüística del andaluz occidental, con especial atención a los rasgos lingüísticos específicos de Cádiz (Romero y Jiménez, 20I6).

\subsection{Fase II: Comprensión}

Para el desarrollo de esta fase, programada con dinámicas de aprendizaje cooperativo, serán necesarias cinco sesiones: tres de la asignatura Lengua Castellana y Literatura y dos de Música. Será imprescindible contar con la mediación docente (Esteban, 2OII). Los productos resultantes se incluirán en el portfolio del proyecto para su posterior evaluación: - Los aspectos lingüísticos se abordarán a través de un taller literario cuyo objetivo será la creación de alegrías de Cádiz respetando sus características formales y cumpliendo sus requisitos temáticos. De nuevo, para escribir alegrías será necesario acudir a modelos, consensuar en grupo las temáticas abordadas, decidir sobre la métrica, etc. Una vez más, el alumnado contará con el Padlet creado ad hoc por los docentes.

- Los aspectos musicales se abordarán a través de un taller musical cuyo objetivo será la experimentación del ritmo propio de las alegrías de Cádiz percutiendo con el propio cuer- 
po y con distintos elementos de percusión, según el desarrollo musical del alumnado. Para lograr interpretar este palo flamenco será necesario realizar distintas audiciones y visionados, analizar la estructura interna de las piezas, leer partituras, etc. El alumnado podrá recurrir a un entorno informacional creado por los docentes en Padlet para encontrar todo lo que desee.

Tras la indagación realizada en los talleres, el alumnado tendrá la posibilidad de acceder a los recursos suficientes y organizar la información que habrá de incluir en el documental: bailaoras singulares, grandes guitarras del flamenco, historia de las alegrías, temáticas características, aspectos formales... La pauta consiste en que los estudiantes utilicen estos contenidos para contextualizar las grabaciones de las alegrías que han escrito, recitado y acompañado rítmicamente.

\section{3·3. Fase III: Práctica}

En esta fase se trata de que el alumnado realice ejercicios, a modo de andamiaje, -desde un planteamiento intensivo- que aseguren que las tareas puedan cumplirse con éxito -desde un enfoque extensivo- (Romero y Trigo, 20I5; Romero y Jiménez, 2OI6). Se ocuparán dos sesiones: una, para Lengua Castellana y Literatura; y otra, para Música. Los ejercicios consistirán en el trabajo de elementos rítmicos musicales: palmeo, percusión, entonación (si procediera en el grupo); y de elementos poéticos como la medida de los versos, los acentos, la sinalefa, la diéresis y la sinéresis. Los ejercicios realizados se incluirán dentro del portfolio del proyecto.

\subsection{Fase IV: Transferencia: creación del storyboard y del montaje.}

En esta fase, que tendrá una duración de cuatro sesiones dentro de la asignatura de Lengua Castellana y Literatura, cada grupo, con toda la información recabada en el proceso, deberá componer el storyboard que servirá de base para finalizar el documental. Esta fase será crucial para el éxito del proyecto (Corpas y Rubio, 2OI7). Es el momento de planificar el texto final: el documental y, por ello, las decisiones tomadas deberán reflexionarse con anterioridad. Cada grupo contará con papel A3 para poder organizar las secuencias y decidir los elementos audiovisuales que se incluirán en cada momento: las imágenes que se desecharán; aquellas que, por el contrario, se incluirán, etc. El papel del docente será fundamental puesto que deberá ir orientando a los grupos para que encaminen sus esfuerzos hacia la elaboración de un buen trabajo. El docente se servirá, para evaluar, de una parrilla de observación (anexo 2) que le servirá, además, para hacer reflexionar al alumnado sobre el mensaje que está construyendo: si es coherente la historia, si se ven claramente los elementos tipográficos, si son todas las partes audibles, si se está cumpliendo con la consigna inicial... 


\section{3·5. Fase V: Evaluación: visionado (coevaluación y heteroevaluación) y selec- ción del documental}

En esta última fase se contará con dos sesiones en la asignatura de Música para la elaboración del documental. Una vez que se ha realizado el storyboard, es el momento de pasar al montaje y la edición del vídeo. Para ello se utilizarán aplicaciones de fácil manejo para los estudiantes. Entre ellas se destacan iMovie para Apple o Story Remix si se utiliza el sistema Android y Touch Cast, compatible con ambos sistemas utilizando una tableta digital como cámara y permitiendo mezclar vídeo con contenido web, delimitar escenarios, insertar elementos multimedia, etc ${ }^{6}$.

Tras el montaje del documental, se empleará una sesión de cada asignatura para la coevaluación y la heteroevaluación (Lorente, 2OII). Se utilizará, para esta ocasión, la rúbrica elaborada para evaluar el proyecto (anexo 3). Dicho proceso consistirá en una exposición oral en la que cada grupo proyectará y comentará su trabajo. A partir de las directrices dadas a través de la rúbrica, se decidirá cuál de los proyectos es el más representativo de la clase y se compartirá con los estudiantes de Aragón pues, como indica Cano (2OI5), el hecho de utilizar una rúbrica para la evaluación es muy conveniente puesto que el alumnado tiene la posibilidad de orientar su proceso creativo.

4. Conclusiones y discusión

A través de este proyecto, hemos contribuido al desarrollo de...

\footnotetext{
${ }^{6}$ En el siguiente enlace se puede encontrar una guía para el manejo de esta aplicación y varios ejemplos de proyectos educativos en los que se utiliza: https://goo.gl/b3Q474
} 


\begin{tabular}{|c|c|}
\hline $\begin{array}{l}\text {.. la competencia matemática, en cien- } \\
\text { cia y tecnología: }\end{array}$ & $\begin{array}{l}\text { porque... } \\
\ldots . \text { los estudiantes recurren a conceptos nu- } \\
\text { méricos para representar elementos musi- } \\
\text { cales. }\end{array}$ \\
\hline ... la competencia lingüística: & $\begin{array}{l}\text {... los estudiantes valoran la importancia } \\
\text { de la escritura para la comunicación; dis- } \\
\text { tinguen modalidades de habla; se expresan } \\
\text { de forma oral en poesía; utilizan el lengua- } \\
\text { je para la composición e interpretación de } \\
\text { alegrías de Cádiz; escriben textos utilizan- } \\
\text { do el registro adecuado y respetan las nor- } \\
\text { mas gramaticales. }\end{array}$ \\
\hline ... la competencia digital: & $\begin{array}{l}\text {.. los estudiantes utilizan recursos tecno- } \\
\text { lógicos para obtener información y crear } \\
\text { contenidos. }\end{array}$ \\
\hline ... la competencia social y cívica: & $\begin{array}{l}\text {.. los estudiantes respetan la modalidad } \\
\text { de habla andaluza y se comunican en con- } \\
\text { textos musicales. }\end{array}$ \\
\hline $\begin{array}{l}\text {.. la competencia en conciencia y expre- } \\
\text { siones culturales: }\end{array}$ & $\begin{array}{l}\text {... los estudiantes reconocen la modalidad } \\
\text { de habla andaluza; conocen los rasgos es- } \\
\text { tructurales de las alegrías de Cádiz; inter- } \\
\text { pretan alegrías de Cádiz y exploran distin- } \\
\text { tos recursos para conocer el concepto de } \\
\text { alegría. }\end{array}$ \\
\hline $\begin{array}{l}\text {... la competencia para aprender a } \\
\text { aprender: }\end{array}$ & $\begin{array}{l}\text {... los estudiantes utilizan la escritura para } \\
\text { adquirir aprendizajes; recurren a los proce- } \\
\text { sos implicados en el aprendizaje (memo- } \\
\text { rización); son capaces de planificar, tex- } \\
\text { tualizar y revisar producciones propias y } \\
\text { ajenas; y organizan sus ideas para producir } \\
\text { textos coherentes y cohesionados. }\end{array}$ \\
\hline $\begin{array}{l}\text {.. la competencia para el sentido de } \\
\text { iniciativa y espíritu emprendedor: }\end{array}$ & $\begin{array}{l}\text {.. los estudiantes utilizan la escritura } \\
\text { como estímulo de desarrollo personal e } \\
\text { indagan sobre el hecho musical utilizando } \\
\text { recursos de las TIC para crear. }\end{array}$ \\
\hline
\end{tabular}

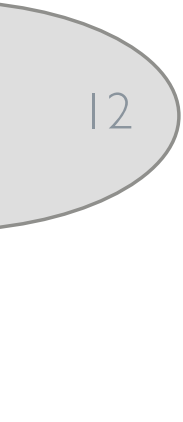

\section{competencia para aprender a aprender:}

... la competencia para el sentido de iniciativa y espíritu emprendedor: 
En el apartado de la introducción destacábamos que la utilización del flamenco como recurso didáctico no supone una novedad. Son numerosas las propuestas que, desde los años ochenta del siglo pasado, han visto la luz en busca de la valoración y difusión del patrimonio cultural andaluz entre los más pequeños.

La normativa educativa en Andalucía prescribe la inclusión del flamenco en las escuelas, desde la Educación Infantil hasta el Bachillerato. Como se ha podido apreciar, son múltiples los recursos institucionales a disposición del profesorado en cuanto a la innovación y a la formación permanente se refiere. No obstante, si analizamos los programas de formación inicial del profesorado en las universidades andaluzas, apreciamos que la difusión del flamenco pasa casi inadvertida: tan solo se realizan leves guiños desde la tradición oral. Aunque estudios, como el de López (2004), muestren algunas experiencias realizadas en la Universidad de Sevilla y que en ciertas universidades, como en la de Cádiz, se hayan creado espacios para la difusión -tanto cultural como formativa- del flamenco, aún es necesario seguir trabajando para que esta presencia no sea tangencial, sino constante en la formación de futuros docentes.

Desde nuestra propuesta queremos plantear que sin formación, a veces, no es posible desarrollar una buena práctica, llevarla a cabo desde la verdadera compresión de la enseñanza. Por ello ponemos a disposición del profesorado -en formación inicial y en ejercicio- un diseño de trabajo funcional, desde la reflexión de los elementos curriculares encaminada a tratar uno de los temas tan recomendados y recomendables como, seamos realistas, ausentes en nuestras aulas: el flamenco. 


\section{Referencias}

En este apartado incluimos la bibliografía utilizada para la redacción de este artículo pero, además, ofrecemos a docentes e investigadores una lista de recursos que pueden encontrarse en red para continuar profundizando en la relación existente entre literatura y flamenco y para llevar el flamenco al aula.

\section{I. Bibliografía}

- Ambròs, A. y Ramos, J. M. (2007). Veamos poesía: leamos imágenes en secundaria. Aula de Innovación Educativa, I65 (pp. 82-96).

- Consejería de Educación de la Junta de Andalucía (2OI4). Orden de 7 de mayo de 20I4, por la que se establecen medidas para la inclusión del Flamenco en el sistema educativo andaluz.

- Consejería de Educación de la Junta de Andalucía (20I4). Instrucciones de 6 de noviembre de 2014 para la celebración del Día del Flamenco, que se conmemora el día i6 de noviembre

- Consejería de Educación de la Junta de Andalucía (20I6). Decreto III/20I6, de I4 de junio, por el que se establece la ordenación y el currículo de la Educación Secundaria Obligatoria en la Comunidad Autónoma de Andalucía.

- Cano, E. (20I5). La rúbrica como instrumento de evaluación de competencias en la Educación Superior ¿uso o abuso?, Profesorado. Revista de Currículum y Formación del Profesorado, I9 (2) (pp. 265-280).

- Corpas, A. (20I7). Sentidos figurados. Una propuesta para la creatividad de la lírica en el IES Vega de Mijas de Las Lagunas (Málaga). Libro Abierto. Disponible en: https://goo.gl/ pMX6ET, consultado el i de enero de 2018.

- Corpas, A. y Rubio, R. (20I7). Expandiendo el aula a través del microblogging. Revista de Estudios Socioeducativos. ReSed, 5 (pp. II9-г29).

- Cruces, C. (2003). Antropología y flamenco. Más allá de la música (II). Sevilla: Signatura.

- De Vicente, M. I. (20I3). Poesía y canción. Una investigación interdisciplinar en Educación Secundaria Obligatoria. Cancelas, L., Jiménez, R., Romero, M. F., Sánchez, S. Aportaciones para una lingüística y literaria en el siglo XXI. Granada: GEU.

- Delgado, B. (2OII). Leyendo el Sur. A propósito del libro Cantes al Amorsillega de Ginés Liébana, Alabe, 3 (pp. I-I9), disponible en: http://www.ual.es/alabe [accedido el 22 de enero de 20I8]. 
- Escamilla, A. (2009). Competencia en tratamiento de la información y digital. Concepto, componentes, relaciones y recursos. MULTIárea Revista de Didáctica, Escuela Universitaria de Magisterio de Ciudad Real, 4. (pp.97-Io8).

- Esteban, M. (2OII). Del “Aprendizaje Basado En Problemas" (ABP) al "Aprendizaje Basado En La Acción" (ABA). Claves para su complementariedad e implementación. REDU. Revista de Docencia Universitaria, 9 (I) (pp. 9I-IOZ).

- Fernández-Fígares, M. D. (1995). Los gitanos: pueblo y cultura. Gaceta de Andalucía. Los gitanos en Andalucía, año IX, número especial.

- Feito, R., (2OIO). De las competencias básicas al currículo integrado. Revista Qurriculum, 23 (pp.55-79)

- Gutiérrez, F. (1990). La copla flamenca y la lírica de tipo popular. Madrid: Cinterco.

- Gutiérrez, R. y Perea, B. (20I5). La Didáctica del Flamenco y su multidisciplinariedad. El proyecto COFLA (Análisis computacional de la música flamenca. DEDiCA. Revista de Educação e Humanidades, 7. (pp. I63-174).

- Julio, F. A. (2OI2). Proyecto 'flamencas' una experiencia didáctico-flamenca en la ESO. Revista de investigación sobre flamenco. La Madrugá, 7. (pp. I35- I56).

- León, C. (1990). Talleres de cultura andaluza: didáctica del flamenco. Sevilla: Dirección General de Renovación Pedagógica y Reforma, Departamento de Cultura Andaluza, Junta de Andalucía.

- Lomas, C. (20IO). Cómo enseñar a hacer cosas con las palabras: Teoría y práctica de la educación lingüística. Barcelona: Paidós.

- López, M. (20IO). La didáctica del flamenco: una aproximación a su historia y algunas propuestas de trabajo. Revista de investigación sobre flamenco. La Madrugá, 3. (pp. I-27).

- Lorente, P. (2OII). Retos de la evaluación en Lengua Castellana y Literatura para el siglo XXI. Tejuelo, II. (pp. IO4-I27).

- Majó, F. (2OIO). Por los proyectos interdisciplinarios competenciales. Aula de Innovación Educativa, I95 (pp.7-II).

- Ministerio de Educación Cultura y Deporte (20I4). R.D. IIO5/20I4, de 26 de diciembre, por el que se establece el currículo básico de la Educación Secundaria Obligatoria y del Bachillerato 
- Navarro, J. L. (i986). Un curso de flamenco para enseñantes. Candil, 46 (pp. $3^{\mathrm{I}-32}$ ).

- Perea, B. (20I4) El flamenco en primaria. Material on-line disponible en https://goo.gl/ Dwkizr, consultado el I3/IO/2OI\%.

- Puig, J.M. y Martín, X. (2007). Competencia en autonomía e iniciativa personal. Madrid: Alianza.

- Romero, M. F. y Jiménez, R. (20I6). La lectura intensiva como estrategia para el desarrollo de habilidades comunicativas entre estudiantes de secundaria de ELE. J. Wilk-Racięska, A. Szyndler, C. Tatoj (eds.), Relecturas y nuevos horizontes en los estudios hispánicos: lingüística y didáctica, Wydawnictwo Uniwersytetu Śląskiego (pp. 339-36I).

- Romero, M. F. y Trigo, E. (20I5). Herramientas para el éxito. Cuadernos de Pedagogía, $45^{8}$ (pp. г6-2I).

- Romero, M. F., Heredia, H. y Ordóñez, A. (20I7). Las TIC como elemento de cohesión en el desarrollo de la oralidad mediante el ABP. Un estudio de caso. @TIC. Revista d'innovació educativa, I9 (pp. 30-38).

- Rossy, H. (ig66). Historia del cante jondo. Madrid: Cresda.

- Sánchez, C. y Navarro, J. L. (I988). Aproximación a una didáctica del flamenco. Sevilla: Junta de Andalucía.

- Trujillo, F. (20I6). Aprendizaje basado en proyectos. Infantil, Primaria y Secundaria. Madrid: Ministerio de Educación Cultura y Deporte.

-Uccella, F. R. (20I3). Manual de patrimonio literario. Espacios, casas-museo y rutas. Gijón: Trea.

- Utrilla, J. (2007). El flamenco se aprende. Teoría didáctica para la enseñanza del flamenco. Córdoba: Toro mítico.

- Zabala, A. y Arnau, L. (2007). 11 ideas clave, cómo aprender y enseñar competencias. Barcelona: Graó.

- Zabala, A. y Arnau, L. (20I4). Métodos para la enseñanza de las competencias. Barcelona: Graó. 


\subsection{Webgrafía. Recursos para el profesorado}

\section{El concepto de alegría:}

- Ortiz, M. (20Iо). Flamenco Viejo. Palos flamencos. Alegrías. [online] Disponible en: https://goo.gl/cA87hQ [accedido el i6 de enero de 20I8].

- Temprano, A. (20I5). Alegrías de Cádiz. Padlet. [online] Disponible en: https://goo.gl/ oWZm3b [accedido el 20 de enero de 20I8].

- YouTube (20I8). Niña Pastori. Cuando miro tus ojos (en directo). [online] Disponible en: https://goo.gl/5DmREk [accedido el I4 de enero de 20I8].

\section{Sobre la relación entre literatura y flamenco:}

-Aguilar, C. (20I3). El Flamenco en la literatura andaluza. [online] Disponible en: https:// goo.gl/pu6dKJ [accedido el I de enero de 20I8].

- Catedraflamencologia.us.es. (2018). Literatura y Flamenco - I Jornadas del ciclo de Literatura y Flamenco celebradas en Sevilla Octubre 20o9. [online] Disponible en: https://goo. $\mathrm{gl} / \mathrm{jkuztN}$ [accedido el ıo de enero de 20I8].

- Flamenco, I. (20I8). II Jornadas `Literatura y Flamenco' | Revista La Flamenca. [online] Revistalaflamenca.com. Disponible en: https://goo.gl/KHw4Fg [accedido el 4 de enero de 20I8].

- Feandalucia.ccoo.es. (2018). Literatura y Flamenco. [online] Disponible en: https://goo. gl/NzQG53 [accedido el i de enero de 20r8].

- López, J. (2018). Flamenco y Literatura. [online] Flamencoyliteratura.blogspot.com.es. Disponible en: https://goo.gl/FJV26G [accedido el 3 de enero de 20I8].

\section{Propuestas y herramientas didácticas para llevar el flamenco al aula:}

- Juntadeandalucia.es. (20I8). Flamenco - Inicio - Consejería de Educación. [online] Disponible en: https://goo.gl/D7XmCY [accedido el I de enero de 20I8].

- Perea, B. (20I8). “El Flamenco en Primaria”. [online] Flamencoprimaria.blogspot.com. es. Disponible en: https://goo.gl/Dwkizr, [accedido el 22 de enero de 20I8].

- Rimador.net. (20r8). Diccionario de rimas Rimador Net, palabras que rimen con lo que quieras. [online] Disponible en: http://www.rimador.net/ [accedido el I de enero de 20I8]. 


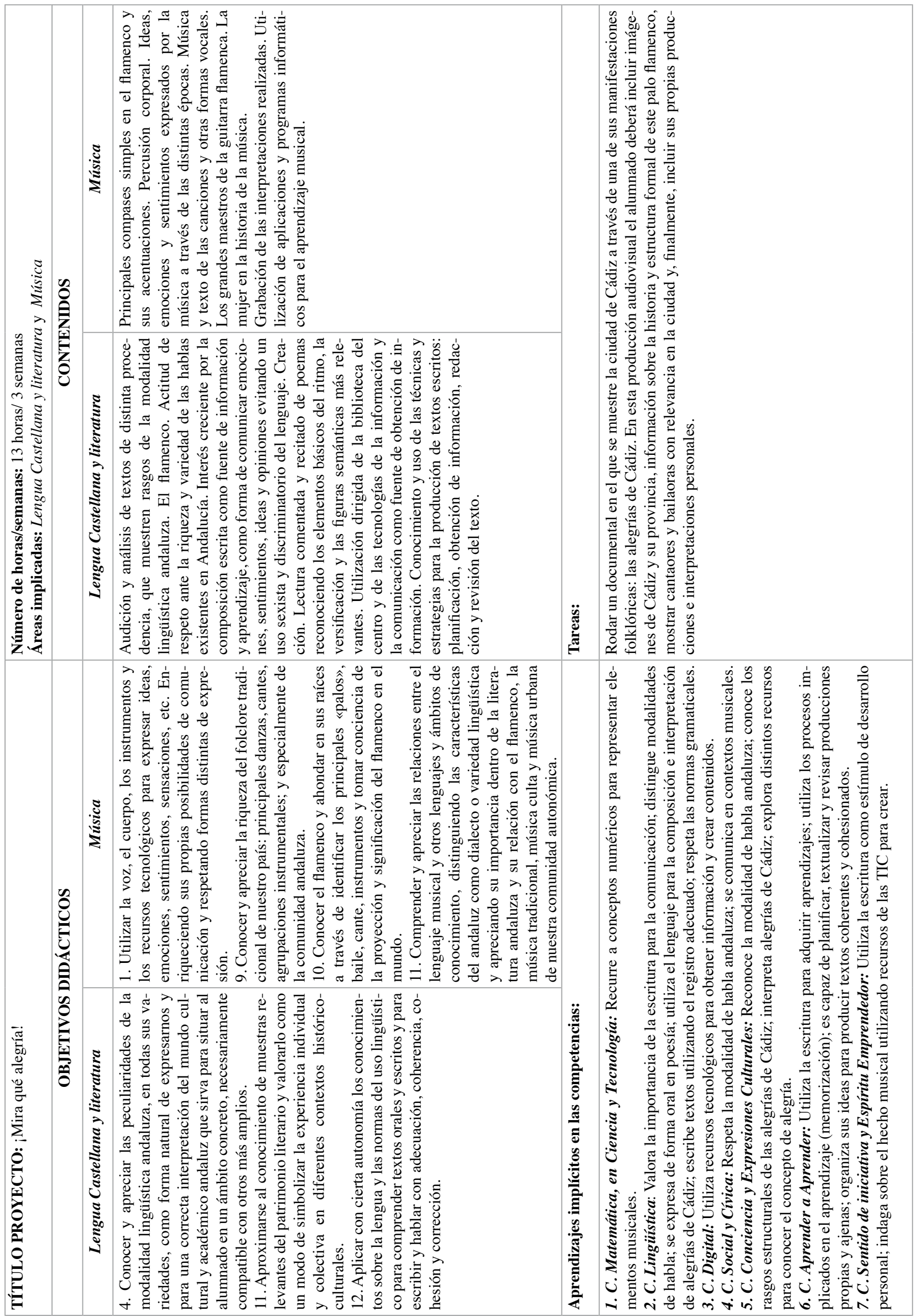




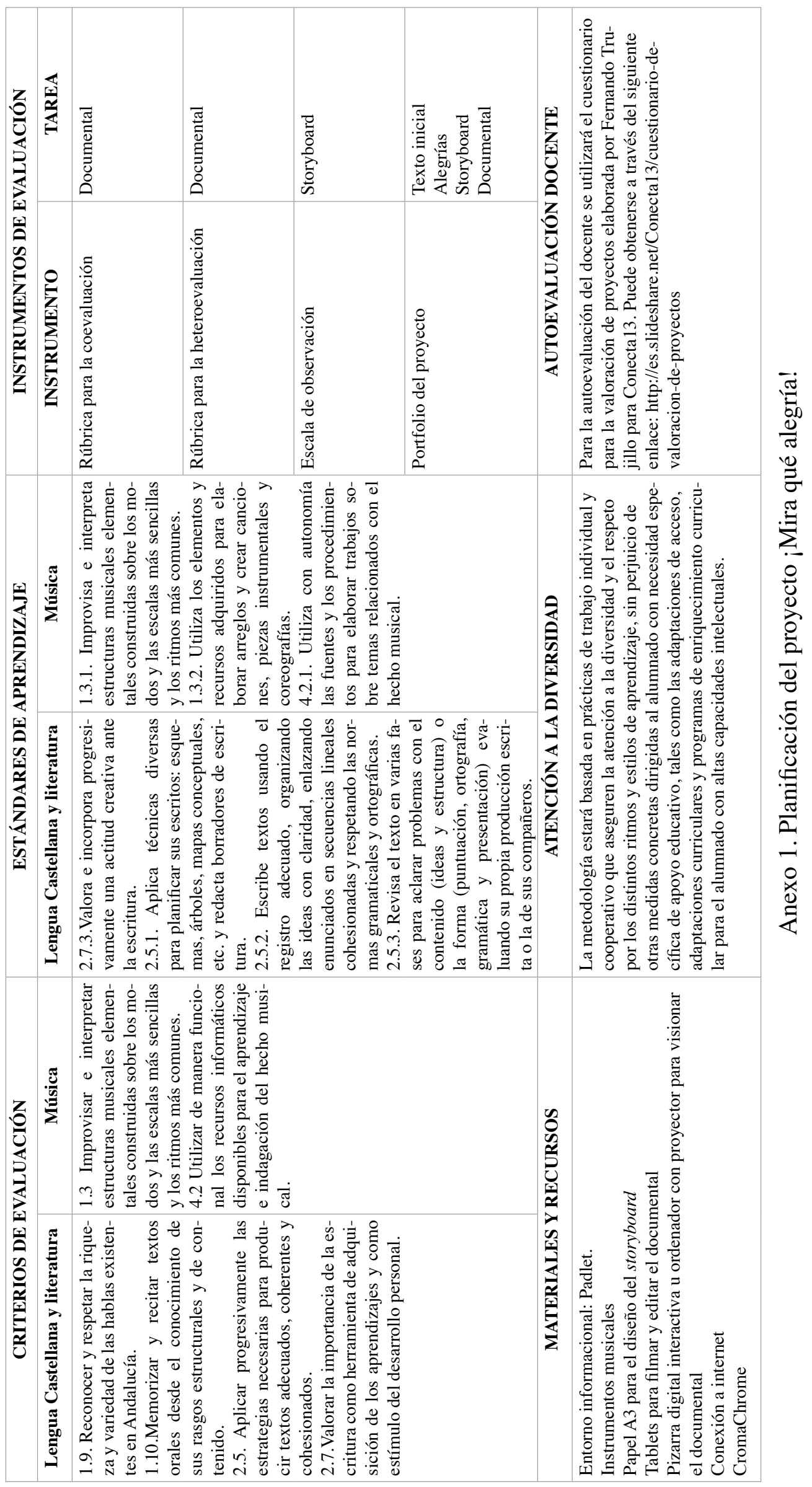




\begin{tabular}{|l|l|l|l|}
\hline \multicolumn{3}{|c|}{ PLANIFICACIÓN DE UNA PRÁCTICA LETRADA: EL STORYBOARD } \\
\hline En cuanto al trabajo en grupo & 1 & 2 & 4 \\
\hline Mantiene una actitud de colaboración con el grupo & & & \\
\hline Aporta ideas y manifiesta iniciativa propia & & & \\
\hline Es respetuoso con las ideas de los demás & 1 & 3 & \\
\hline En cuanto al proceso de planificación del documental & & & \\
\hline Realiza un esquema para plasmar las ideas & & & \\
\hline Organiza las secuencias bajo un hilo conductor & & & \\
\hline Incorpora elementos procedentes de diversas fuentes & & & \\
\hline Planifica los elementos audiovisuales: fondos, música... & & & \\
\hline En cuanto a los textos seleccionados & & & \\
\hline Respeta los criterios formales de las alegrías de Cádiz & & & \\
\hline Inserta sus producciones en las temáticas prefijadas & & & \\
\hline Aporta variedad de textos propios y ajenos & & & \\
\hline En cuanto al texto “narrador” del documental & & & \\
\hline Realiza un esquema para planificar las ideas & & & \\
\hline Tiene claro los objetivos antes de escribir el texto & & & \\
\hline Selecciona información pertinente & & & \\
\hline Determina la estructura (introducción-nudo-conclusión) & & & \\
\hline
\end{tabular}

Anexo 2. Parrilla de observación 


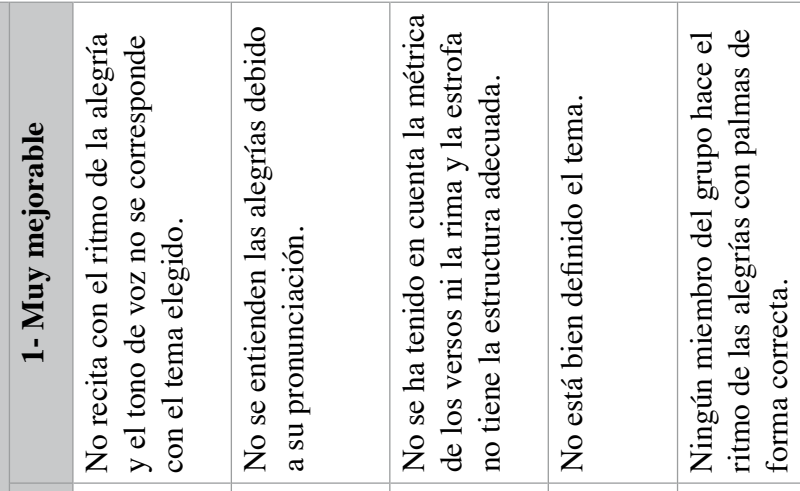

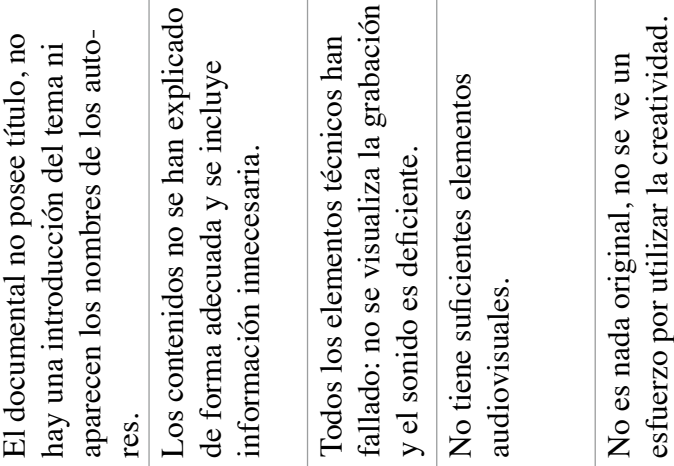

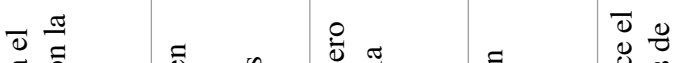

म

ìn

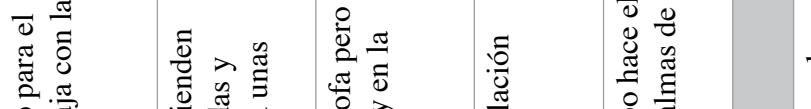

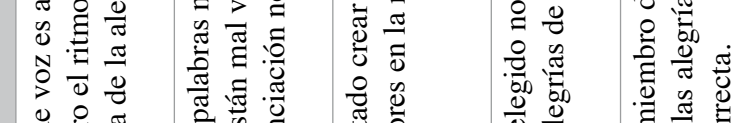

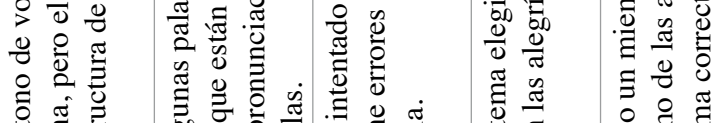

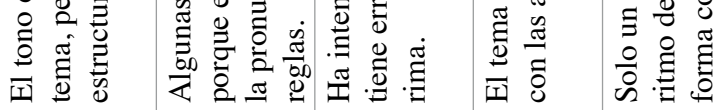

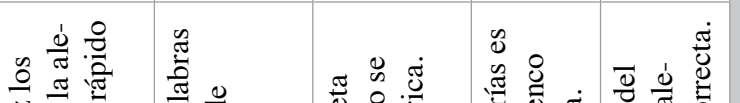

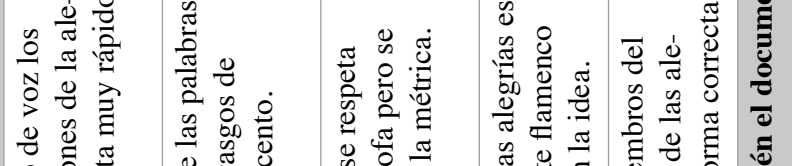

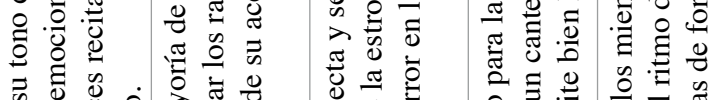

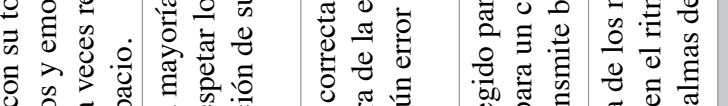

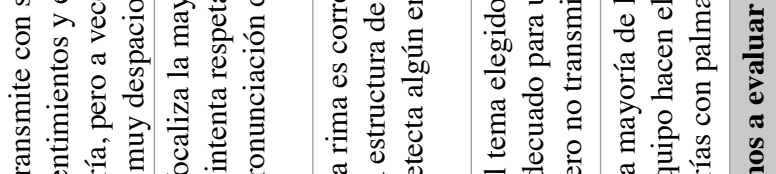

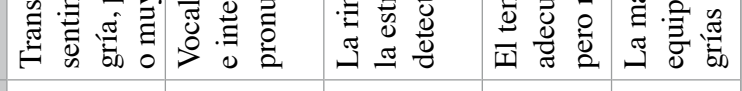

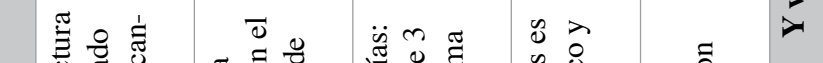

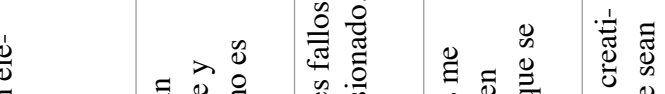

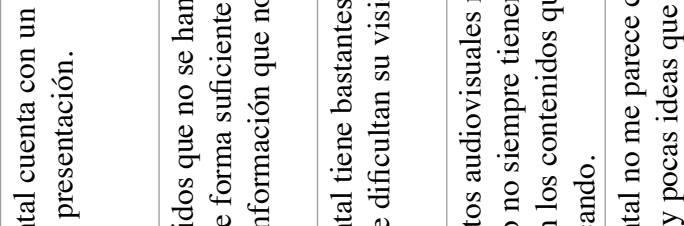

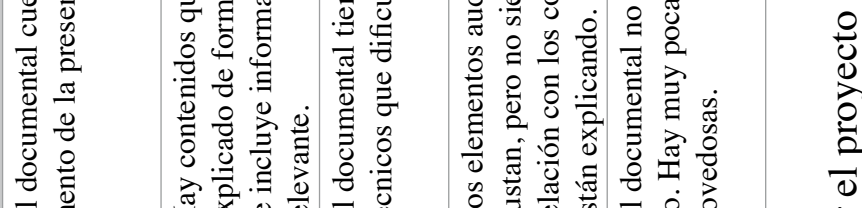

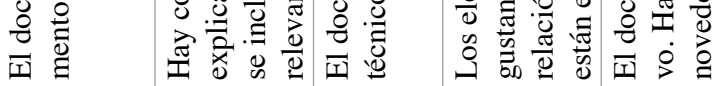

\begin{tabular}{|c|c|c|c|c|}
\hline & & & & \\
\hline 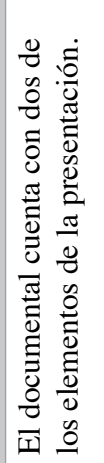 & 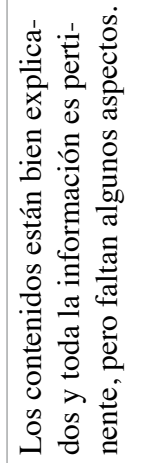 & 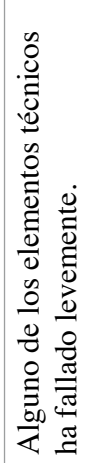 & 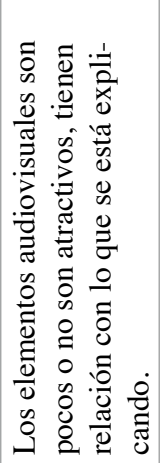 & 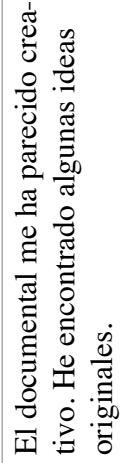 \\
\hline
\end{tabular}

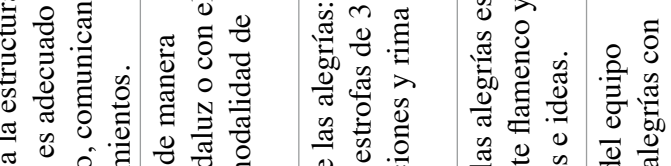

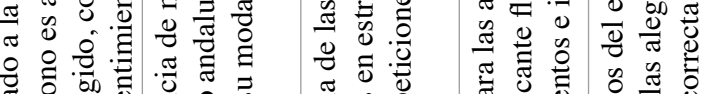

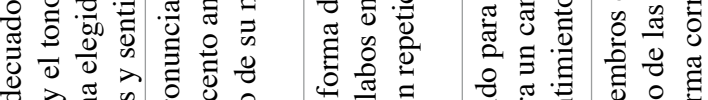

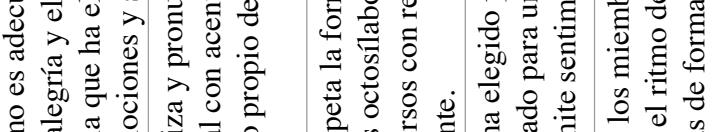

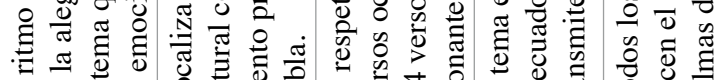

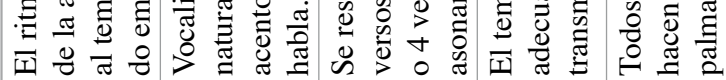

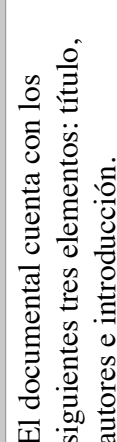

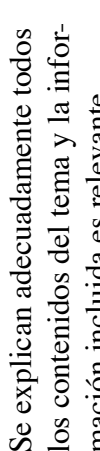
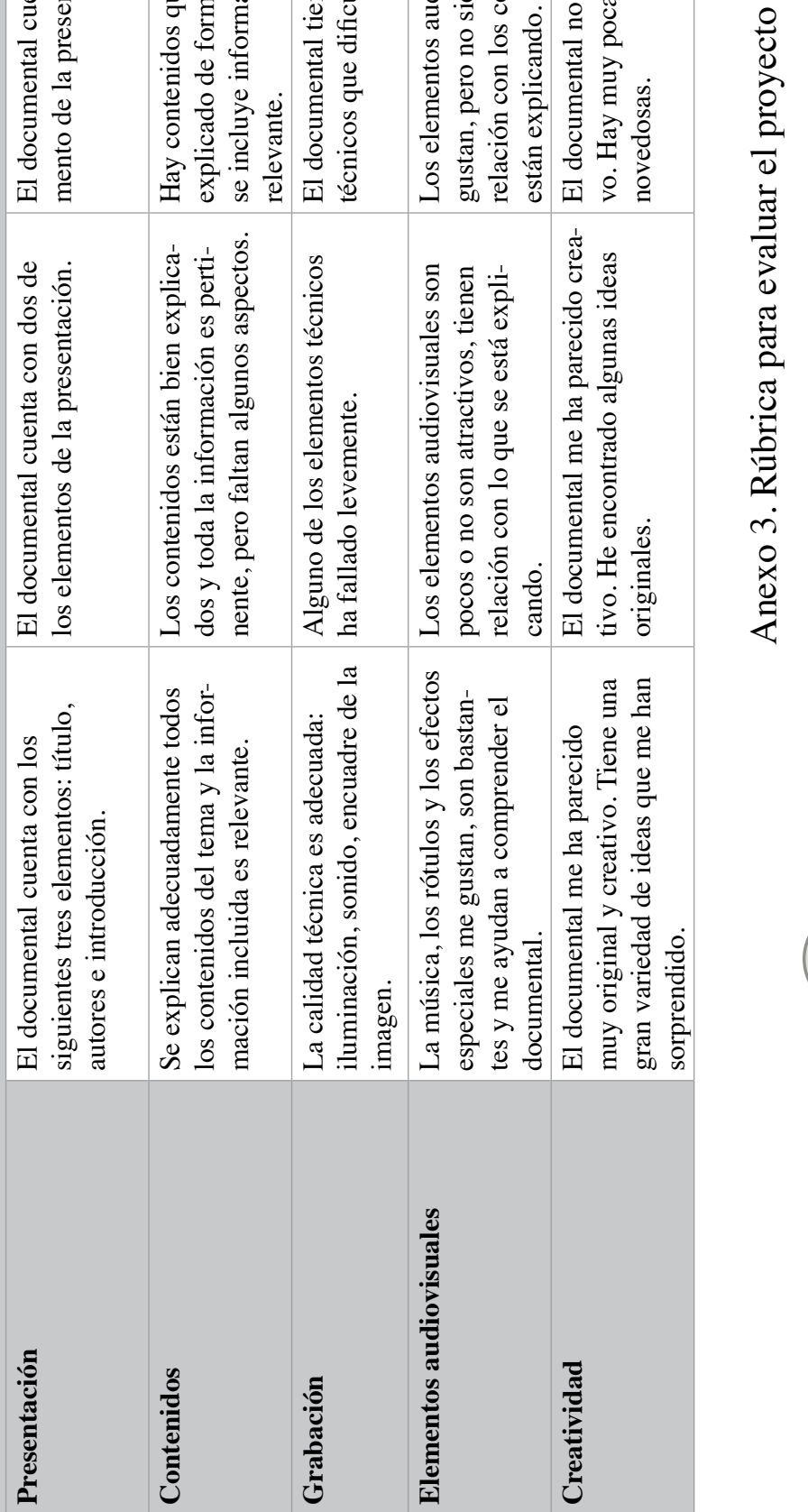